\title{
In Vivo Optical Microendoscopy for Imaging Cells Lying Deep within Live Tissue
}

\author{
Robert P.J. Barretto and Mark J. Schnitzer
}

\begin{abstract}
Although in vivo microscopy has been pivotal in enabling studies of neuronal structure and function in the intact mammalian brain, conventional intravital microscopy has generally been limited to superficial brain areas such as the olfactory bulb, the neocortex, or the cerebellar cortex. For imaging cells in deeper areas, this article discusses in vivo optical microendoscopy using gradient refractive index (GRIN) microlenses that can be inserted into tissue. Our general methodology is broadly applicable to many deep brain regions and areas of the body. Microendoscopes are available in a wide variety of optical designs, allowing imaging across a range of spatial scales and with spatial resolution that can now closely approach that offered by standard water-immersion microscope objectives. The incorporation of microendoscope probes into portable miniaturized microscopes allows imaging in freely behaving animals. When combined with the broad sets of available fluorescent markers, animal preparations, and genetically modified mice, microendoscopic methods enable sophisticated experimental designs for probing how cellular characteristics may underlie or reflect animal behavior and life experience, in healthy animals and animal models of disease.
\end{abstract}

Recent strides in intravital light microscopy have enabled seminal studies of both neuronal structure and dynamics in the intact mammalian brain (Gobel and Helmchen 2007; Kerr and Denk 2008; Rochefort et al. 2008; Holtmaat et al. 2009; Holtmaat and Svoboda 2009; Wilt et al. 2009). Applications of two-photon microscopy in awake but head-restrained animals have even permitted $\mathrm{Ca}^{2+}$-imaging studies during active animal behavior (Dombeck et al. 2007; Mukamel et al. 2009; Nimmerjahn et al. 2009). However, photon scattering limits the optical penetration of light microscopy into tissue, restricting the utility of conventional intravital microscopy to superficial tissue areas such as the olfactory bulb, the neocortex, and the cerebellar cortex (Helmchen and Denk 2005; Wilt et al. 2009). Penetration depths are typically limited to $\sim 50-100 \mu \mathrm{m}$ with epifluorescence microscopy and $\sim 500-700 \mu \mathrm{m}$ with conventional two-photon microscopy.

To extend the microscope's penetration depth into tissue, a range of innovative optical strategies has been experimentally explored in the last few years (Helmchen and Denk 2005; Wilt et al. 2009). Here, we describe one of these approaches: optical microendoscopy (Jung and Schnitzer 2003; Jung et al. 2004; Levene et al. 2004), which can penetrate the furthest of these and reach $>1 \mathrm{~cm}$ into tissue (Llewellyn et al. 2008) via the use of needle-like micro-optical probes. These probes typically act like an optical relay and can be inserted into tissue. Subject to some optical constraints discussed below, the length of the probe can be tailored to the anatomical depth of the tissue under examination. Optical microendoscopy provides spatial resolution that can approach that of a conventional water-

Adapted from Imaging: A Laboratory Manual (ed. Yuste). CSHL Press, Cold Spring Harbor, NY, USA, 2010.

(C) 2012 Cold Spring Harbor Laboratory Press

Cite this article as Cold Spring Harb Protoc; 2012; doi:10.1101/pdb.top071464 
immersion objective lens (Barretto et al. 2009); is compatible for use with multiple contrast modalities including epifluorescence, two-photon excited fluorescence, and second-harmonic generation (Mehta et al. 2004; Flusberg et al. 2005); and has been used in both live mice and humans (Llewellyn et al. 2008; Wilt et al. 2009). In this article, we present optical considerations in the choice of a microendoscope probe and modifications to the upright light microscope that facilitate microendoscopy. Methods for imaging cellular characteristics in the intact mammalian brain are provided in In Vivo Microendoscopy of the Hippocampus (Barretto and Schnitzer 2012). The technique is also compatible for use with portable miniaturized microscopes (Gobel et al. 2004; Engelbrecht et al. 2008; Flusberg et al. 2008) that are based on micro-optics and enable imaging in freely behaving mice (Flusberg et al. 2008).

\section{IMAGING SETUP}

Microscope Body

Nearly any upright microscope that has infinity optics and has already been adapted for in vivo imaging (e.g., Ultima IV, Prairie Technologies, Inc.) can readily be used for microendoscopy. There are two main options for how the microendoscope probe can be held (Barretto et al. 2009), one of which requires custom modifications to the microscope.

In the simpler approach, the microendoscope probe is held by its insertion into the animal subject, instead of being coupled mechanically to the body of the microscope. When the animal and the microendoscope probe are positioned correctly, the microendoscope relays the focal plane of the microscope objective into deep tissue, with a demagnification or magnification that depends on the optical details of the probe. This approach has the advantage of not requiring any alterations to the microscope but the disadvantage that any fine adjustments of the microendoscope relative to the tissue are not automatically referenced to the optical axis.

In an alternative approach, the microendoscope probe is mounted on the microscope's focusing unit, which is modified to permit two modes of fine focal adjustment (Fig. 1A,C). The first mode adjusts the position of the microscope objective lens relative to the microendoscope probe. The second mode moves the objective lens and the microendoscope probe in tandem, permitting the microendoscope to be inserted into tissue without affecting the optical coupling to the objective lens. Both modes can be motorized. To grip the microendoscope probe on its sides, we use a two-pronged pincer holder (e.g., Thorlabs, Inc., Micro-V-Clamp) (Fig. 1C). This holder is attached to a miniature probe clamp (e.g., Siskiyou, Inc., MXC-2.5) that can be rotated about its long axis and swung in and out of the optical pathway. By adjusting the two angular degrees of freedom of the probe clamp, we align the microendoscope with the optical axis (Barretto et al. 2009). Adjustments in the axial position of the objective lens while keeping the microendoscope fixed are performed using a stepper motor (Sutter Instruments, MP-285) mounted on the microscope's nosepiece. These adjustments modify the intermediate plane at which the illumination is focused above the microlens, leading to corresponding focal adjustments in the specimen. The microendoscope and objective lens are moved in tandem using the microscope's normal focusing actuator (Fig. 1C).

\section{Microendoscope Probes}

Microendoscope probes can be customized for specific applications (Fig. 1D), and distinct values of the probes' basic optical parameters are preferred in different situations. For example, some optical designs are better suited for examining subcellular features such as dendritic structures, whereas other designs are preferred for wide-field $\mathrm{Ca}^{2+}$ imaging of neuronal dynamics. In our own work, we have explored three main types of optical designs. The first design involves a single GRIN lens (Fig. 1D, top left) that provides low magnification and a large field of view. The second design involves a GRIN lens attached in series to a high-numerical-aperture (NA) $(\sim 0.65-0.82)$ plano-convex microlens (Barretto 
A

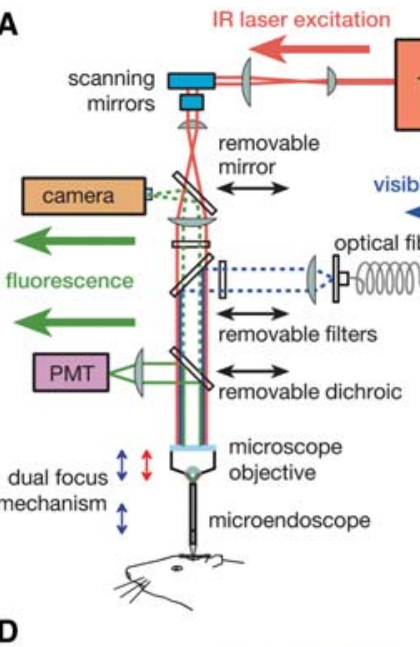

0.19 pitch singlet

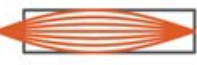

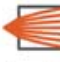

FIGURE 1. Methodologies for in vivo optical microendoscopy. (A) Optical schematic of an upright microscope modified to permit both one- and two-photon fluorescence microendoscopy. For two-photon imaging, the beam from an ultrashort-pulsed infrared (IR) Ti:sapphire laser is scanned within the focal plane of the microscope objective. By adjusting the axial separation between the objective and the microendoscope (red arrow of the dual-focus mechanism; see also C), this focal plane of the microscope objective is also set to the microendoscope's back focal plane. Another focal adjustment (blue arrows of the dual mechanism) is used to lower the objective and the microendoscope in tandem toward the animal. For one-photon imaging, a mercury $(\mathrm{Hg})$ arc lamp provides illumination. In both imaging modes, fluorescence emissions route back through the microendoscope and to either a camera or a photomultiplier tube (PMT) for one- or two-photon imaging, respectively. (B) Photographs of the tips of a 0.5-mm-diameter microendoscope of doublet design (top) and a 0.8-mm-outer-diameter glass capillary guide tube (bottom) into which this microendoscope can be inserted. The relay of the microendoscope is coated black. A glass coverslip is attached to the tip of the guide tube. The guide tube facilitates the rapid exchange of microendoscopes without perturbation to the underlying tissue. Scale bar, $1 \mathrm{~mm}$. $(C)$ The microscope objective and the microendoscope probe are mounted on a pair of cascaded focusing actuators that provide dual-focus capability. This allows the objective to be moved either alone (red arrow) or together with the microendoscope (blue arrow). The microendoscope can also be swung out of the optical axis (green arrow) to permit conventional microscopy. (D) Optical ray diagrams for sample microendoscopes of the singlet GRIN (top left), compound plano-convex and GRIN (top right), and GRIN doublet (bottom) types. Scale bar, $1 \mathrm{~mm}$.

et al. 2009) (Fig. 1D, top right); this combination can provide superior light collection and diffractionlimited resolution but has a smaller field of view. The third design has a GRIN relay lens coupled to a GRIN objective (Fig. 1D, bottom), allowing longer probe designs ( $>5 \mathrm{~mm}$ ) and intermediate-sized fields of view (Jung et al. 2004; Levene et al. 2004).

Microendoscope probes of all three types (Fig. 1D, Table 1) can generally be conceptualized as consisting of two optical components in series: an infinity micro-objective that focuses illumination to the specimen and collects emission photons, combined with a micro-optical relay lens that receives focused illumination from the microscope and also focuses the sample's emissions to the front focal plane of the upright microscope's objective lens (Fig. 1A,C). In singlet GRIN lenses, both of these functions occur within a single optical element; in GRIN doublet probes or in high-resolution probes, the jobs of the objective and the relay are accomplished by two micro-optical entities attached in series. In epifluorescence microendoscopy, the relay microlens projects a real image of the sample to the microscope objective's focal plane. Table 1 presents optical parameters for some microendoscopes, with each of the three major types represented. Below, we consider these parameters in further detail. For mathematical formulas to guide optical design, see Jung et al. (2004); with these equations, researchers can design probes to custom specifications and have them fabricated commercially. 
TABLE 1. Characteristics of sample microendoscope probes

\begin{tabular}{|c|c|c|c|c|c|c|}
\hline Microendoscope type & $\begin{array}{l}\text { Diameter } \\
(\mathrm{mm})\end{array}$ & $\begin{array}{l}\text { Length } \\
(\mathrm{mm})\end{array}$ & $\begin{array}{l}\text { Usable field } \\
\text { of view }(\mu \mathrm{m})\end{array}$ & $\begin{array}{c}\text { Lateral } \\
\text { magnification) } \\
(\times)\end{array}$ & $\begin{array}{c}\text { Two-photon } \\
\text { lateral resolution } \\
(\mathrm{FWHM}, \mu \mathrm{m})\end{array}$ & $\mathrm{NA}$ \\
\hline Doublet (0.75/0.21 pitch) & 1.0 & 20.6 & 275 & 2.52 & 0.9 & 0.49 \\
\hline Doublet (1.25/0.19 pitch) & 0.5 & 16.4 & 130 & 2.48 & 1.0 & 0.48 \\
\hline Doublet (1.75/0.16 pitch) & 0.35 & 15.8 & 75 & 2.69 & 1.2 & 0.45 \\
\hline Singlet (0.46 pitch) & 1.0 & 4.4 & 700 & 0.97 & 0.9 & 0.49 \\
\hline Singlet (0.94 pitch) & 0.5 & 4.3 & 350 & 0.92 & 1.0 & 0.47 \\
\hline $\begin{array}{l}\text { GRIN/plano-convex doublet } \\
\text { (BK7 plano-convex lens) }\end{array}$ & 1.0 & 3.7 & 120 & 1.41 & 0.8 & 0.65 \\
\hline $\begin{array}{l}\text { GRIN/plano-convex doublet } \\
\text { (LaSFN9 plano-convex lens) }\end{array}$ & 1.0 & 4.0 & 75 & 1.86 & 0.6 & 0.82 \\
\hline
\end{tabular}

To facilitate comparisons between parameter values, each microendoscope listed has an optical working distance of $250 \mu \mathrm{m}$. The lateral resolution of two-photon imaging is given for 920-nm illumination.

FWHM, full width at half-maximum; NA, numerical aperture; GRIN, gradient refractive index.

\section{Microendoscope Diameter}

Microendoscope probes with diameters ranging from 0.35 to $2.8 \mathrm{~mm}$ are commercially available (e.g., from GRINTECH GmbH); our laboratory most commonly uses 0.35-, 0.5-, and 1.0-mm sizes. For a given NA value, the smaller diameter probes (e.g., 0.35 or $0.5 \mathrm{~mm}$ ) offer resolution and magnification values comparable to those of wider diameter probes. However, the wider probes of the same NA will generally have longer working distances to the sample and broader fields of view. In addition, smaller diameter probes are more fragile. Encasing these probes in thin-walled stainless-steel hypodermic sheaths will make them more robust. Probes as thin as $0.35 \mathrm{~mm}$ in diameter have been successfully applied for high resolution in vivo laser-scanning imaging, including in humans (Llewellyn et al. 2008).

\section{Microendoscope Length}

The lengths of microendoscope probes are typically designed to meet the mechanical constraints posed by the depth of the tissue under examination and the surgical preparation. The probe length should be sufficient to guide photons from the specimen plane lying deep within the tissue to an unobstructed intermediate focal plane that is also the focal plane of the microscope objective (Fig. 1A). It is the length of the relay microlens that is typically adjusted in applications requiring imaging at substantial tissue depths.

Valid lengths for the relay microlens are calculated by first determining the pitch length of its glass GRIN substrate (Jung et al. 2004). Within a paraxial approximation, light rays propagate down the optical axis of the microendoscope along a trajectory for which the rays' radial distance from the axis varies as a sinusoidal function of the distance propagated axially (Fig. 1D). One pitch length is defined as the length of the GRIN substrate within which a ray will propagate a full sinusoidal cycle. This length depends on the radially varying refractive index profile of the GRIN material. Cylindrical rods of this material can then be cut to various lengths measured in units of the pitch length. GRIN lenses of integral or half-integral pitch - that is, $1 / 2,1,3 / 2$ pitch, etc.-refocus light rays emanating from a single focus on one side of the lens to another focal spot on the opposite side of the lens. By comparison, 1/4-pitch lenses-or 3/4-pitch lenses, 5/4-pitch lenses, etc.-are infinity lenses that focus collimated rays entering one side of the microlens to a focal spot on the lens's opposing side (see the 0.75-pitch relay in Fig. 1D, bottom). Longer microendoscope probes can be designed by adding multiple 1/2-pitch lengths to the relay lens as necessary. Such additions extend the probe's length without altering the NA, the magnification, the field of view, or the working distance. However, probes of longer length often suffer from poorer optical resolution caused by the accumulation of spherical aberrations over multiple half-pitch lengths of the GRIN substrate. 


\section{Optical Working Distance to the Specimen}

The working distance to the specimen is set for a GRIN objective lens by the degree to which the objective is slightly shorter than a 1/4-pitch design (see the 0.19-pitch singlet and the 0.22-pitch objective in Fig. 1D). An objective of shorter pitch has a longer working distance, but the objective's $\mathrm{NA}$ is reduced. Typical values of working distance range from $0 \mu \mathrm{m}$ to $800 \mu \mathrm{m}$. In one-photon fluorescence imaging, light scattering precludes efficient imaging beyond $\sim 100 \mu \mathrm{m}$ from the tip of the endoscope (Flusberg et al. 2008), so the working distance will be relatively short. By comparison, in two-photon imaging, microendoscopy can be performed up to $\sim 650 \mu \mathrm{m}$ into tissue beyond the probe tip, which generally necessitates a design of longer working distance.

Although the focal plane can be adjusted to a depth other than the working distance, microendoscopes are often designed to have minimal optical aberrations at their specified working distance. In particular, the high-resolution GRIN/plano-convex compound lenses (Fig. 1D, top right) are designed so that aberrations from the objective component are compensated at a specific working distance by an appropriate choice of the GRIN relay's radial refractive index profile (Barretto et al. 2009). High-resolution experiments should, thus, be performed with the tissue of interest located at the designed working distance. However, for imaging experiments that permit modest degradation in resolution, it is convenient to design the optical working distance to be a few hundred micrometers longer than what will be used for the experiment. This choice ensures that neither the plane of laser scanning in two-photon imaging nor the intermediate real image in one-photon imaging is located at external glass surfaces of the microendoscope, where surface imperfections can degrade image quality.

\section{Microscope Objectives for Optical Coupling to the Microendoscope Probe}

\section{Microscope Objective Magnification}

In applications requiring large fields of view, the magnification of the microscope objective should suffice to permit imaging of the entire top surface of the microendoscope probe. For example, a typical $10 \times$ objective has a sufficient field of view to image the entire aperture of a 1-mm-diameter microendoscope probe. Other parameters of the entire optical system must also suffice to image this entire aperture. For example, in one-photon microendoscopy, the camera chip must be sufficiently wide; and in two-photon microendoscopy, the range of laser scanning must be sufficiently broad to sample the entire face of a 1-mm-diameter microendoscope probe.

\section{Microscope Objective NA}

To achieve high-resolution imaging, the NA of the microscope objective should be higher than that of the microendoscope probe's relay lens. In one-photon imaging, this condition ensures that the microscope objective captures the full NA of fluorescence emissions exiting the microendoscope's relay lens, thereby preserving signal power as well as image resolution. In two-photon imaging, this condition ensures that the laser illumination fills the back aperture of the probe's objective lens, typically located at the boundary between the micro-objective and the relay (Fig. 1D) and thereby uses the full NA of the microendoscope's objective in focusing the laser beam at the specimen plane. A portion of the laser illumination will be lost, however, because the NA of the beam striking the relay lens is higher than the NA that the relay can accept.

Imaging Parameters

Detailed imaging parameters for one-photon and two-photon imaging are provided in In Vivo Microendoscopy of the Hippocampus (Barretto and Schnitzer 2012). 


\section{CONCLUSION}

Microendoscopy is a useful technique for expanding the range of tissues accessible to cellular level imaging in live animals or humans. Microendoscope probe designs can be customized to accommodate a wide range of optical requirements. Overall, microendoscopy opens a broad set of possibilities for imaging cells in brain areas outside the reach of conventional light microscopy, for basic research purposes, studies of animal disease models, or testing of new therapeutics.

\section{ACKNOWLEDGMENTS}

This work was supported by the Stanford Biophysics training grant to R.P.J.B. from the U.S. National Institutes of Health and research funding provided to M.J.S. under the National Institute on Drug Abuse Cutting-Edge Basic Research Awards (NIDA CEBRA) DA017895, the National Institute of Neurological Disorders and Stroke (NINDS) R01NS050533, and the National Cancer Institute (NCI) P50CA114747. We thank our collaborators Bernhard Messerschmidt of Grintech GmbH and Tony Ko, Juergen C. Jung, Alessio Attardo, Yaniv Ziv, Michael Llewellyn, Scott Delp, George Capps, Alison Waters, Tammy J. Wang, and Lawrence Recht of Stanford University for their contributions to the methodologies summarized here.

\section{REFERENCES}

Barretto RPJ, Schnitzer MJ. 2012. In vivo microendoscopy of the hippocampus. Cold Spring Harb Protoc doi: 10.1101/pdb.prot071472.

Barretto RP, Messerschmidt B, Schnitzer MJ. 2009. In vivo fluorescence imaging with high-resolution microlenses. Nat Methods 6: 511-512.

Dombeck DA, Khabbaz AN, Collman F, Adelman TL, Tank DW. 2007. Imaging large-scale neural activity with cellular resolution in awake, mobile mice. Neuron 56: 43-57.

Engelbrecht CJ, Johnston RS, Seibel EJ, Helmchen F. 2008. Ultra-compact fiberoptic two-photon microscope for functional fluorescence imaging in vivo. Opt Express 16: 5556-5564.

Flusberg BA, Cocker ED, Piyawattanametha W, Jung JC, Cheung EL, Schnitzer MJ. 2005. Fiber-optic fluorescence imaging. Nat Methods 2: 941-950.

Flusberg BA, Nimmerjahn A, Cocker ED, Mukamel EA, Barretto RP, Ko TH, Burns LD, Jung JC, Schnitzer MJ. 2008. High-speed, miniaturized fluorescence microscopy in freely moving mice. Nat Methods 5: 935-938.

Gobel W, Helmchen F. 2007. In vivo calcium imaging of neural network function. Physiology 22: 358-365.

Gobel W, Kerr JN, Nimmerjahn A, Helmchen F. 2004. Miniaturized twophoton microscope based on a flexible coherent fiber bundle and a gradient-index lens objective. Opt Lett 29: 2521-2523.

Helmchen F, Denk W. 2005. Deep tissue two-photon microscopy. Nat Methods 2: 932-940.

Holtmaat A, Svoboda K. 2009. Experience-dependent structural synaptic plasticity in the mammalian brain. Nat Rev Neurosci 10: 647-658.

Holtmaat A, Bonhoeffer T, Chow DK, Chuckowree J, De Paola V, Hofer SB, Hubener M, Keck T, Knott G, Lee WC, et al. 2009. Long-term, high- resolution imaging in the mouse neocortex through a chronic cranial window. Nat Protoc 4: 1128-1144.

Jung JC, Schnitzer MJ. 2003. Multiphoton endoscopy. Opt Lett 28: 902-904.

Jung JC, Mehta AD, Aksay E, Stepnoski R, Schnitzer MJ. 2004. In vivo mammalian brain imaging using one- and two-photon fluorescence microendoscopy. J Neurophysiol 92: 3121-3133.

Kerr JN, Denk W. 2008. Imaging in vivo: Watching the brain in action. Nat Rev Neurosci 9: 195-205.

Levene MJ, Dombeck DA, Kasischke KA, Molloy RP, Webb WW. 2004. In vivo multiphoton microscopy of deep brain tissue. J Neurophysiol 91: 1908-1912.

Llewellyn ME, Barretto RP, Delp SL, Schnitzer MJ. 2008. Minimally invasive highspeed imaging of sarcomere contractile dynamics in mice and humans. Nature 454: 784-788.

Mehta AD, Jung JC, Flusberg BA, Schnitzer MJ. 2004. Fiber optic in vivo imaging in the mammalian nervous system. Curr Opin Neurobiol 14: 617-628.

Mukamel EA, Nimmerjahn A, Schnitzer MJ. 2009. Automated analysis of cellular signals from large-scale calcium imaging data. Neuron 63: 747-760.

Nimmerjahn A, Mukamel EA, Schnitzer MJ. 2009. Motor behavior activates Bergmann glial networks. Neuron 62: 400-412.

Rochefort NL, Jia H, Konnerth A. 2008. Calcium imaging in the living brain: Prospects for molecular medicine. Trends Mol Med 14: 389-399.

Wilt BA, Burns LD, Wei Ho ET, Ghosh KK, Mukamel EA, Schnitzer MJ. 2009. Advances in light microscopy for neuroscience. Annu Rev Neurosci 32: 435-506. 


\section{In Vivo Optical Microendoscopy for Imaging Cells Lying Deep within Live Tissue}

Robert P.J. Barretto and Mark J. Schnitzer

Cold Spring Harb Protoc; doi: 10.1101/pdb.top071464

\begin{tabular}{cc}
$\begin{array}{c}\text { Email Alerting } \\
\text { Service }\end{array}$ & Receive free email alerts when new articles cite this article - click here. \\
\hline $\begin{array}{c}\text { Subject } \\
\text { Categories }\end{array}$ & $\begin{array}{c}\text { Browse articles on similar topics from Cold Spring Harbor Protocols. } \\
\text { Fluorescence (517 articles) } \\
\text { Fluorescence, general (341 articles) } \\
\text { Imaging for Neuroscience (342 articles) } \\
\text { In Vivo Imaging (334 articles) } \\
\text { In Vivo Imaging, general (168 articles) } \\
\text { Multi-Photon Microscopy (103 articles) }\end{array}$ \\
\hline
\end{tabular}

